\title{
Андерсоновская локализация в синтетической фотонной решетке за счет случайного распределения коэффициента деления
}

\author{
А.В. Паньков", И.Д. Ватник, Д.В. Чуркин \\ Новосибирский государственный университет \\ *E-mail: dabrdadub@mail.ru
}

DOI:10.31868/RFL2018.98-99

За последние несколько десятилетий локализация света привлекла к себе большое количество внимания. Эффект андерсоновской локализации, который изначально был сформулирован для электронов в неупорядоченных системах [1], был затем теоретически и экспериментально продемонстрирован для света в различных фотонных системах: в разупорядоченных двумерных фотонных решетках [2], в синтетических фотонных решетках [3], и даже в биологических наноструктурах натурального шелка [4].

В данной работе численно изучается локализация в синтетической фотонной решетке (СФР) - системе, состоящей из двух оптоволоконных колец различной длины, соединенных настраиваемым оптоволоконным делителем (Рис. 1а). Из-за разницы в длине петель, импульс, помещенный в одну из них, создает последовательность импульсов в каждой из петель, которые интерферируют при каждом обороте, тем самым создавая комплексную картину эволюции. Можно показать, что эволюция импульсов в такой системе эквивалентна эволюции света в одномерной ячеистой фотонной решетке.

Ранее нами уже был экспериментально продемонстрирован эффект андерсоновской локализации в такой решетке, возникающий при внесении случайного набега фаз при движении по решетке [3]. В данной работе в качестве источника беспорядка выступает оптоволоконный делитель с настраиваемым коэффициентом деления $\theta$, а не фазовая модуляция.

Случайное распределение коэффициента деления определялось как $\theta_{n}=$ $\pi / 4 \pm \operatorname{rand}\left(\Delta \theta_{\max }\right)$, где $\pi / 4$ соответствует делителю $50 \% / 50 \%$, функция $\operatorname{rand}(\mathrm{x})$ дает случайное число в диапазоне от 0 до $\mathrm{x}$, а величина $\Delta \theta_{\max }$ определяет силу беспорядка и варьирует в пределах от 0 до $\pi / 4$.

Было изучено влияние случайно распределенного коэффициента деления $\theta_{n}$ на эволюцию импульса, введенного в короткую петлю СФР. Для этого мы проводили расчет эволюции системы, и усредняли ее по 10000 реализациям случайного распределения коэффициента $\theta_{n}$. Результаты расчета демонстрируют наличие андерсоновской локализации (Рис.1б). Для количественного определения уровня локализации был посчитан коэффициент заполнения $P(m)=\sum_{n}\left(\left|U_{n}^{m}\right|^{2}\right)^{2} / \sum_{n}\left|U_{n}^{m}\right|^{4}$. Чем шире волновой пакет $U_{n}^{m}$, тем выше коэффициент заполнения. Этот параметр также усреднен по ансамблю из 10000 реализаций для различных сил беспорядка (Рис. 1в). Числа заполнения выходят на постоянную величину уже спустя менее 100 обходов, что говорит о наличии андерсоновской локализации.

Дополнительно нами были посчитаны усредненные по 2000 реализациям обратные коэффициенты заполнения $(1 / P(m))$ и их флуктуации $\delta(1 / P(m))$ на фиксированном обходе $\mathrm{m}=1000$ в зависимости от плавно возрастающей силы беспорядка $\Delta \theta_{\max }$. В результате мы получили квазилинейный рост как обратного 
коэффициента заполнения, так и его флуктуации (Рис. 1г), что также является подтверждением наличия андерсоновской локализации в такой системе [5].

a)
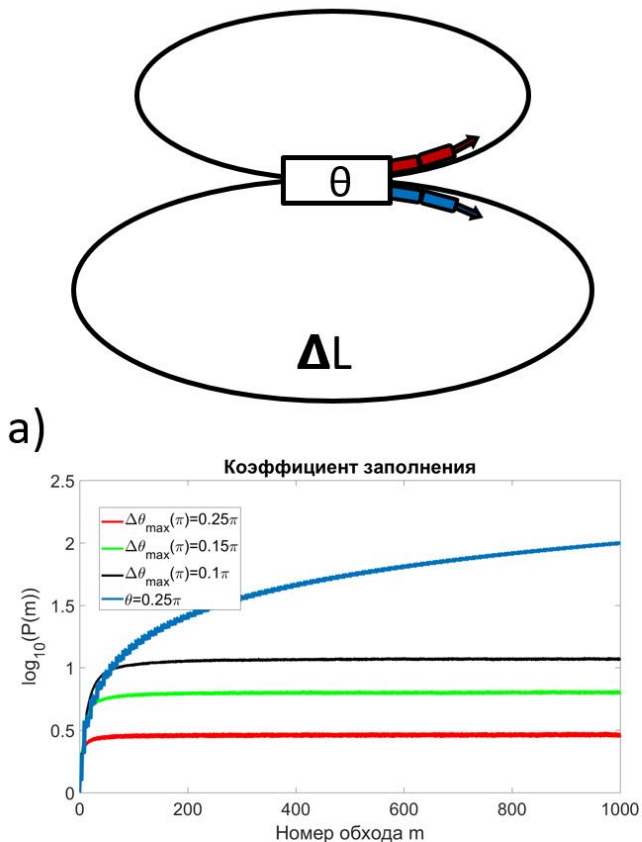

B)

Рис.1. а) Синтетическая фотонная решетка б) Эволюция импульсов с синтетической фотонной решетке со случайными изменениями коэффициента деления с силой беспорядка $\Delta \theta_{\max }=0.25 \pi$ в) Коэффициент заполнения в зависимости от номера обхода $\mathrm{m}$ при различной силе беспорядка $\Delta \theta_{\max }$ г) Усредненные по 2000 реализациям обратные коэффициенты заполнения и их флуктуации на обходе $\mathrm{m}=1000$ для различной силы беспорядка $\Delta \theta_{\max }$.

Работа выполнена при поддержке гранта РНФ (16-12-10402).

\section{Литература}

[1] Anderson, P. W., Physical review, 109(5), 1492 (1958)

[2] Schwartz, Tal, et al., Nature 446 52-55 (2007)

[3] Vatnik, Ilya D., et al., Scientific reports 7.1, 4301 (2017)

[4] Choi, Seung Ho, et al., Nature communications 9.1, 452 (2018)

[5] Segev, M.,, et al., Nature photonics 7, 197 (2013) 